\title{
Ética y ciencias de la vida: un análisis crítico de las concepciones acerca del valor de lo vivo presentes en la biología*
}

Ethics and Life Sciences: a critical analysis of the conceptions about the value of the living in Biology

Constanza Alexandra Rendón ${ }^{\dagger}$

Gabriela Klier

\begin{abstract}
Resumen
En el presente artículo analizamos las concepciones acerca del valor de lo vivo actualmente presentes en diferentes áreas de la biología (biología molecular, genética, fisiología y biología de la conservación). La hipótesis que guió este trabajo es que existen distintos supuestos acerca del valor de lo vivo en diferentes subdisciplinas biológicas, lo cual podría implicar conflictos valorativos al seno de la biología. A partir del análisis realizado hallamos una amplia valoración productiva de los organismos en todas las áreas (además de su valoración como herramientas para obtener conocimiento), mientras que sólo en el caso de la biología de la conservación encontramos el reconocimiento del valor intrínseco de lo vivo. Hallamos también que esta última subdisciplina asigna valor intrínseco a especies y ecosistemas, negando tal valor a los organismos individuales. Esta atribución de valor intrínseco a entidades biológicas diferentes a los organismos contribuiría a evitar conflictos valorativos con otros campos de estudio en los cuales prima la valoración utilitaria de los organismos. Poner en cuestión los valores y supuestos que subyacen a las prácticas científicas resulta central en el contexto actual en el que las ciencias de la vida participan de diversas controversias éticas.
\end{abstract}

Palabras clave: valor de lo vivo - biología - ética

\begin{abstract}
In this paper we analyze the conceptions regarding the value of the living in different biological fields (Molecular Biology, Genetics, Physiology and Conservation Biology). The hypothesis that guided this study is that there might be different assumptions about the value of the living in distinct biological sub-disciplines, and this situation could generate value conflicts within biology. We found that the productive value of organisms (in addition to their value as tools for obtaining knowledge) is widely acknowledged in all the sub-disciplines, while the intrinsic value of the living is only recognized in the area of Conservation Biology. This field of study assigns intrinsic value to species and ecosystems, rejecting intrinsic value of individual organisms. This negation of organisms' intrinsic value would contribute to avoid conflicts with other biological fields in which utilitarian value of the living prevail. Inquiring about values and assumptions underlying scientific practices is crucial in the current context in which life sciences participate in diverse ethical controversies.
\end{abstract}

Keywords: value of the living - biology - ethics

\footnotetext{
* Recibido: 30 de Marzo de 2016. Aceptado con revisiones: 5 de Diciembre de 2016.

† Instituto de Filosofía "Dr. Alejandro Korn", Facultad de Filosofía y Letras, Universidad de Buenos Aires. Para contactar con el autor, por favor, escribir a: constanzarendon@yahoo.com.

* Departamento de Ecología, Genética y Evolución, Facultad de Ciencias Exactas y Naturales, Universidad de Buenos Aires. Para contactar con el autor, por favor, escribir a: gabrielaklier@gmail.com.

Metatheoria 8(2)(2018): 135-143. ISSN 1853-2322.

(c) Editorial de la Universidad Nacional de Tres de Febrero. Publicado en la República Argentina.
} 


\section{Introducción. Ciencia y valores. Objetivos y metodología}

En el presente trabajo nos proponemos analizar qué concepciones acerca del valor de lo vivo se encuentran presentes actualmente en la biología. La pregunta que nos guía puede enmarcarse entonces en la exploración de la relación entre la ética y la biología. La relación entre ética y ciencia ha sido analizada e interpretada desde diversas perspectivas. En particular, varios autores han destacado que la visión canónica tradicionalmente sostenida desde la ciencia ha sido la de considerar a la ética como separada del ámbito científico (Marcusse 1985, Heler 1996, Linares 2008). Tal separación se ha vinculado con la aceptación de la objetividad y neutralidad del conocimiento científico (Heler 1996, Rodríguez Alcázar 1997, Kincaid, Dupré \& Wylie 2007, Chakravartty 2015). En función de tales características, los denominados valores no epistémicos quedarían (o deberían quedar) por fuera del ámbito de producción del conocimiento científico (Kincaid, Dupré \& Wylie 2007, Elliott \& McKaughan 2014).

Desde ya, múltiples elementos involucrados en la visión de la ciencia como objetiva y neutral han sido cuestionados y criticados desde diversos enfoques, principalmente en los campos de la historia, la filosofía y la sociología de la ciencia (Marcuse 1985, Heler 1996, García Palacios et al. 2001, Echeverría 2003, Kincaid, Dupré \& Wylie 2007, Linares 2008, Reiss \& Sprenger 2014, Chakravartty 2015). En términos generales, tales críticas parten de considerar a la ciencia como una práctica social e históricamente situada, la cual entabla vínculos complejos con diferentes actores sociales y con los sistemas productivos, involucrando múltiples fines y valores. Sin embargo, no es evidente en qué medida esta visión ha sido igualmente reconocida en otros ámbitos, particularmente, en las propias comunidades científicas.

En este contexto el objetivo general del presente trabajo es indagar qué tipo de valoración de lo vivo puede reconocerse en la biología actualmente. Para ello resulta necesario considerar que la biología es un campo sumamente amplio y diverso, conformado por una multiplicidad de subdisciplinas. En este sentido, cabe mencionar que las reflexiones en torno al valor de la diversidad biológica han cobrado importancia recientemente en el campo de la biología de la conservación, en función de los objetivos propios de esta área (tema que desarrollaremos en la sección siguiente). Sin embargo, la cuestión más general del valor de lo vivo no ha sido igualmente discutida en otros campos de la biología. Por lo tanto, el objetivo específico del presente trabajo es analizar coincidencias y diferencias en el valor dado a lo vivo en diferentes subdisciplinas biológicas. Nuestra hipótesis es que podrían existir distintos supuestos respecto del valor de lo vivo en diferentes áreas, lo cual podría implicar tensiones o conflictos valorativos entre diferentes campos de estudio al seno de la biología.

Para abordar este objetivo la metodología utilizada consistió, en primer lugar, en rastrear nociones acerca del valor de lo vivo en libros de texto de referencia de diferentes campos de estudio de la biología. Los textos seleccionados son considerados como canónicos por las propias comunidades científicas y empleados en la enseñanza universitaria de la biología en las principales universidades de diversos países. La elección de este tipo de fuentes se fundamenta en varios elementos, principalmente, en el hecho de que es en este tipo de bibliografía en la cual suelen plasmarse las ideas y supuestos mayormente aceptados por las comunidades científicas. Además, nociones tales como las que nos proponemos analizar aquí (compromisos éticos y valorativos) no suelen encontrarse explicitadas en otro tipo de fuentes tales como artículos de revistas científicas. ${ }^{1}$ Seleccionamos entonces textos

\footnotetext{
${ }^{1}$ Más específicamente, hemos realizado un análisis de carácter cualitativo y exploratorio de los contenidos de los libros de texto. La descripción y discusión de este tipo de metodologías (así como de sus limitaciones y críticas) pueden encontrarse en diversos trabajos (por ejemplo, Fernández Chaves 2002, López Noguero 2002, Piñuel 2002). Si bien una de las principales críticas que suele realizarse a este tipo de metodologías es la escasa representatividad que los análisis cualitativos pueden tener, creemos que esta limitación puede ser en parte relativizada en el caso del presente trabajo debido a que los textos utilizados en la formación universitaria de los científicos (y considerados como canónicos por las propias comunidades científicas) presentan una notable homogeneidad entre las diversas universidades del mundo. Desde ya las comunidades científicas pueden ser igualmente diversas en los supuestos y posturas éticas y valorativas presentes, y tal diversidad puede no encontrarse representada en los libros de texto canónicos de las subdisciplinas científicas.
} 
correspondientes a áreas diversas de la biología, especializadas en el estudio de diferentes niveles de organización y de distintos aspectos de lo vivo: biología molecular, genética, fisiología, y biología de la conservación. A lo largo de los libros de texto seleccionados rastreamos nociones explícitas acerca del valor otorgado a lo vivo, ya sea de manera general o a alguna entidad biológica en particular (e.g. genes, ecosistemas, etc.), o bien aseveraciones que nos permitieran inferir algún tipo de valor dado a tales entidades. Finalmente, a los fines de ordenar el análisis realizado, clasificamos las valoraciones halladas en tres categorías, definiendo así tres tipos de valor atribuido a lo vivo, a saber:

- Valor productivo: refiere al valor de lo vivo en tanto producto en sí mismo o como medio para producir bienes o servicios, útiles para el humano.

- Valor gnoseológico: alude a la atribución de valor a lo vivo en tanto objeto de conocimiento y/o herramienta para obtener conocimiento.

- Valor intrínseco: valor de lo vivo en sí mismo, independientemente de cualquier utilidad que pudiera tener para los humanos. ${ }^{2}$

A continuación (Sección 2) presentaremos los resultados del análisis realizado para cada campo de estudio. Finalmente, en la Sección 3, compararemos los resultados encontrados en las diferentes áreas analizadas y finalizaremos con algunas conclusiones acerca de los supuestos valorativos presentes en la biología actual.

\section{Análisis de las concepciones acerca del valor de lo vivo en diferentes campos de la biología}

\subsection{Biología molecular y genética}

En esta sección analizaremos las concepciones acerca del valor de lo vivo en dos de las principales subdisciplinas biológicas que abordan los niveles inferiores de organización: la biología molecular y la genética. En primer lugar, encontramos que en la bibliografía principal correspondiente a estas áreas las referencias a cuestiones éticas y valorativas son escasas (Lewin 1999, Alberts et al. 2008, Griffiths et al. 2010). A pesar de ello, en el caso de la genética se vislumbra una valoración fundamentalmente productiva de los organismos. Se destaca en este sentido el progreso tecnológico que la manipulación genética de los organismos habría permitido. Por ejemplo:

Muchos beneficios para la humanidad han resultado de aplicaciones de la genética en la medicina, la agricultura y la industria. Consideremos la agricultura moderna. La mayor parte de los cereales y animales de granja de hoy se relacionan escasamente con las especies salvajes halladas en la naturaleza porque sus genomas han sido extensamente modificados por programas de cruzas sistemáticas. Aunque este proceso de selección comenzó hace siglos, la genética tradicional y la molecular lo han optimizado para producir variedades valiosas en un tiempo mucho más corto. Ahora casi no hay ningún límite a las combinaciones de genes posibles que pueden producirse (Griffiths et al. 2010, p. 23)

Por otra parte, también hallamos en estos campos de estudio la valoración gnoseológica de los organismos. En este sentido suele destacarse en la bibliografía la importancia de los denominados 'organismos modelo' para el desarrollo de la 'revolución' genética y molecular del siglo XX (Alberts et al. 2008, Griffiths et al. 2010). Una interesante cita en uno de los principales libros de texto de genética ilustra la valoración gnoseológica de los organismos modelo:

Sin embargo creemos que el análisis de esta bibliografía permite una primera aproximación general al estudio de algunos aspectos del ethos científico (Maingueneau 1996).

2 La noción de valor intrínseco ha sido sumamente discutida (Callicott 1990, Gudynas 2009). Mientras que algunas definiciones refieren a la valoración de lo vivo sólo por ser viviente (Taylor 1986), otras enfatizan la valoración intrínseca como independiente de la utilidad para el ser humano. Por ejemplo, en el contexto de la ética ambiental, Gudynas define los valores intrínsecos como "valores presentes en elementos del ambiente o en seres vivos, independientes de los seres humanos" (Gudynas 2015, p. 28). Una de las principales críticas que suele presentarse a esta noción de valor intrínseco es que, en tanto el acto de valorar se considera como fundamentalmente humano, no sería posible pensar en una valoración por fuera de nuestra propia subjetividad (Gudynas 2015). 


\begin{abstract}
Otra ventaja para un investigador individual que pertenece a tal comunidad [una comunidad que trabaja con un organismo modelo específico] es que él o ella puede desarrollar 'un sentimiento por el organismo' (una frase de la genetista del maíz y ganadora del premio nobel Bárbara McClintock). Esta idea es difícil de expresar, pero implica una comprensión general de un organismo. Ningún proceso de lo vivo tiene lugar en aislamiento, y conocer de manera general un organismo suele ser beneficioso para tratar de entender un proceso e interpretarlo en su contexto apropiado (Griffiths et al. 2010, p. 731).
\end{abstract}

Así el 'sentimiento por el organismo' es interpretado en términos puramente gnoseológicos, sin considerar otro tipo de valores que puedan sustentarlo. En relación con esto, no hallamos en la bibliografía aseveraciones que apunten a la valoración intrínseca de los organismos utilizados en la experimentación y el desarrollo científico y tecnológico. En este sentido cabe destacar que las secciones dedicadas a los organismos modelo en los textos versan sobre los aspectos técnicos de su utilización, sin menciones a pautas éticas relativas a la experimentación con animales. En resumen, a partir de nuestro análisis hallamos que las subdisciplinas abocadas al estudio de los niveles inferiores de organización destacan el valor productivo y gnoseológico de los organismos, reconociéndolos como importantes herramientas de investigación y producción, sin considerar su valor intrínseco. ${ }^{3}$ Analizaremos a continuación el campo de estudio de la fisiología.

\title{
2.2. Fisiología
}

Nos centraremos en esta sección en el campo de la fisiología animal. Incluimos en nuestra investigación bibliografía correspondiente a la fisiología médica (Boron \& Boulpaep 2012) y a la neurobiología (Purves et al. 2012). A partir de nuestro análisis hallamos en estos campos la valoración productiva y gnoseológica de los organismos. Uno de los tópicos en cuyo tratamiento se vislumbran tales valores es la experimentación con animales, práctica destacada como la metodología principal de la fisiología. En algunos textos encontramos incluso apartados que buscan responder a los crecientes cuestionamientos de la experimentación animal planteados por grupos defensores de los derechos de los animales (Randall, Burggren \& French 2001, Hickman, Roberts \& Larsen 2006). En tales secciones se asume la defensa de la experimentación animal a partir de posturas claramente utilitaristas, destacando los beneficios que esta práctica ha generado para los seres humanos. Por ejemplo:

Las ediciones anteriores de este libro asumieron que los beneficios de la experimentación animal eran comprendidos por todos. Esta suposición ya no puede hacerse. A pesar de los beneficios generales que la experimentación animal ha producido para todos los humanos existen controversias acerca de la ética de utilizar animales en experimentos (Randall, Burggren \& French 2001, p. 12).

Estas secciones son dedicadas luego a enumerar tales beneficios (fundamentalmente aquellos vinculados a la medicina), omitiendo la discusión de los valores que sustentan las diferentes posturas respecto de la experimentación con animales (Randall, Burggren \& French 2001, Hickman, Roberts \& Larsen 2006). ${ }^{4}$ Cabe destacar además la asimetría planteada entre la valoración dada a los seres humanos y aquella otorgada a otros animales en estos apartados (tal asimetría constituye un elemento fundamental en la definición de valor productivo enunciada en la Sección 1 del presente artículo). Si bien no se explicita en los textos en qué elementos se basaría esta asimetría, sí se resalta la importancia vital de la experimentación con animales en función de que sería éticamente inaceptable realizar ciertos experimentos en seres humanos (Randall, Burggren \& French 2001, Hickman, Roberts \& Larsen 2006, Purves et al. 2012). Los textos destacan así la valoración productiva de los organismos por constituir herramientas fundamentales para obtener productos farmacéuticos y tecnologías médicas.

\footnotetext{
${ }^{3}$ Cabe mencionar que encontramos cierta diversidad en la bibliografía correspondiente al campo de la genética en relación con el tratamiento de cuestiones éticas y valorativas. En este sentido hallamos que uno de los textos de referencia del área plantea diversas cuestiones éticas (Klug et al. 2012). Sin embargo, tales discusiones versan sobre tecnologías aplicadas a los seres humanos (tales como el denominado 'mejoramiento humano', la terapia génica, la experimentación con células troncales, entre otras). Se infiere entonces también en este caso la asimetría entre el planteo de cuestiones éticas involucradas en las prácticas científicas que atañen directamente a los seres humanos y aquellas que involucran a otros seres vivos.

${ }^{4}$ Las posturas contrarias a la experimentación con animales no son explicadas en los textos, sino simplemente caracterizadas como inexactas o sentimentalmente distorsionadas (Hickman, Roberts \& Larsen 2006).
} 
Este tipo de valoración se evidencia también en el caso de las aplicaciones agrícolas y ganaderas del conocimiento derivado de la fisiología, el cual contribuiría a mejorar la calidad de los productos extraídos de los animales (Randall, Burggren \& French 2001).

Además, como mencionamos, encontramos en la fisiología la valoración gnoseológica de los organismos como herramientas imprescindibles para la generación de conocimiento acerca del ser humano. También se destaca en la bibliografía la importancia de determinados organismos utilizados como modelos para el estudio de funciones fisiológicas específicas debido a características particulares de cada especie (Randall, Burggren \& French 2001, Hickman, Roberts \& Larsen 2006, Purves et al. 2012). La valoración gnoseológica de los organismos resulta sumamente evidente en el caso de la neurobiología, campo de la fisiología que se basa en el estudio del sistema nervioso de diversos animales para comprender el funcionamiento del cerebro humano y las bases biológicas de trastornos neurológicos y psiquiátricos (Purves et al. 2012). ${ }^{5}$

Finalmente cabe mencionar que la bibliografía parece negar el valor intrínseco de los organismos. Tal negación queda evidenciada en los mencionados apartados dedicados a la experimentación con animales. En esas secciones se explicita el apoyo de los científicos a las normas bioéticas para la experimentación animal contraponiendo esta postura a la defensa de los 'derechos intrínsecos' de los animales (Randall, Burggren \& French 2001, Hickman, Roberts \& Larsen 2006). ${ }^{6}$ Además en las secciones dedicadas al armado de diseños experimentales no se hace referencia a pautas éticas que deban ser tenidas en cuenta en la programación o ejecución de los procedimientos que involucran animales. En conclusión, el análisis realizado indicaría que la fisiología otorga valor productivo y gnoseológico a los organismos, y niega su valor intrínseco.

\subsection{Biología de la conservación}

La biología de la conservación ha surgido como un campo de estudio cuyo objetivo central es contribuir a evitar la pérdida de diversidad biológica (Lindenmayer \& Burgman 2005). En función de tal objetivo, en esta área se ha desarrollado cierta reflexión en torno al valor de lo vivo y a los fundamentos de su conservación. En este sentido encontramos que en los principales textos del área el valor intrínseco de la biodiversidad es considerado como uno de los fundamentos para su conservación (Lindenmayer y Burgman 2005, Groom, Meffe \& Carrol 2006, Hunter \& Gibbs 2007, Van Dyke 2008). Pero más allá de esta aseveración de carácter general, y en función de nuestro objetivo, nos preguntamos cuáles son específicamente las entidades biológicas a las cuales se otorga valor intrínseco en la bibliografía. Hallamos que el valor intrínseco es principalmente atribuido a las especies biológicas (Lindenmayer \& Burgman 2005, Hunter \& Gibbs 2007, Van Dyke 2008) y a los ecosistemas (Groom, Meffe \& Carrol 2006, Van Dyke 2008). Por ejemplo, Hunter y Gibbs (2007) adjudican valor intrínseco a las especies, fundamentando así la necesidad de su conservación:

Uno puede argumentar que cada especie tiene valor intrínseco; en otras palabras, su importancia es independiente de su relación con las personas y con otras especies. Desde esta perspectiva, los conservacionistas suelen evaluar la importancia de una especie según su grado de amenaza (Hunter \& Gibbs 2007, p. 63).

Según estos autores el valor intrínseco de los ecosistemas dependería del valor de las especies que lo habiten: "los ecosistemas tienen valor ya que las especies que contienen tienen valor" (Hunter \& Gibbs

\footnotetext{
${ }^{5}$ Cabe mencionar que la extrapolación de los resultados obtenidos a partir de la experimentación con animales a los humanos ha sido cuestionada en diversos casos, particularmente en el campo de la neurobiología (de Boo \& Knight 2008, Bunner, Chandrasekera \& Barnard 2014, Barbero 2014).

${ }^{6}$ Además, las pautas bioéticas relativas al 'bienestar' de los animales utilizados en experimentación solo son mencionadas brevemente y de manera incompleta en algunos textos (Randall, Burggren \& French 2001, Purves et al. 2012). Las normas más extensamente difundidas en todo el mundo se basan en el denominado 'principio de las tres erres' el cual establece la necesidad de reducir la cantidad de animales utilizados, refinar las técnicas de experimentación a los fines de provocar la menor cantidad de sufrimiento posible, y desarrollar técnicas y métodos de investigación que reemplacen el uso de animales (de Martínez et al. 2007, de Boo \& Knight 2008). Randall y colaboradores (2001) mencionan los dos primeros principios, pero omiten el tercero.
} 
2007, p. 70). ${ }^{7}$ Sin embargo, como mencionamos, otros autores destacan la necesidad de conservar ecosistemas sosteniendo su valor intrínseco. Por ejemplo: "[la meta de la biología de la conservación es] conservar ecosistemas naturales y procesos biológicos, los cuales son considerados como intrínsecamente valiosos para los biólogos de la conservación" (Groom, Meffe \& Carrol 2006, p. 23). Desde esta perspectiva se destaca la conservación del 'teatro ecológico', metáfora que enfatiza la importancia de las relaciones de las especies entre sí y con su entorno (Groom, Meffe \& Carrol 2006).

En relación con lo anterior resulta interesante notar que la atribución de valor intrínseco a los ecosistemas y a las especies implica en muchos casos negar este tipo de valoración a los organismos individuales. Esta negación se ve reflejada, por ejemplo, en las metodologías propuestas para el manejo de las denominadas 'especies invasoras'. Tal como se describe en la mayor parte de los textos analizados, el manejo de estas especies implica generalmente su erradicación mediante remoción, control químico o biológico, o bien su uso directo en función de algún beneficio económico (Van Dyke 2008). Además, en la presentación de estas prácticas de manejo no se reconoce ningún problema ético involucrado en el exterminio de los organismos (Lindenmayer \& Burgman 2005, Groom, Meffe \& Carrol 2006, Hunter \& Gibbs 2007, Van Dyke 2008). Por lo tanto parece asumirse que el valor de las especies y ecosistemas 'nativos' prima por sobre la vida y el valor intrínseco de los organismos individuales. ${ }^{8}$

Por otra parte, también encontramos en la bibliografía la atribución de valor productivo a lo vivo como fundamento para su conservación. Este tipo de valoración se expresa en las posturas utilitaristas que destacan los beneficios de la conservación de la biodiversidad para el humano. En esta línea se encuentran las propuestas que subrayan los servicios ecosistémicos, esto es, las diversas utilidades (en forma de bienes o servicios) que proveen los ecosistemas a las sociedades humanas (Lindenmayer \& Burgman 2005, Groom, Meffe \& Carrol 2006, Hunter \& Gibbs 2007, Van Dyke 2008). Entre tales servicios se destaca la utilidad de entidades biológicas particulares, principalmente especies (por ejemplo, especies de insectos que funcionan como polinizadores) y genes (fundamentalmente por las posibles aplicaciones medicinales de las moléculas codificadas) (Groom, Meffe \& Carrol 2006).

En síntesis, hallamos en la biología de la conservación posturas explícitas que destacan principalmente el valor productivo de diversas entidades biológicas y el valor intrínseco de especies y ecosistemas. A continuación, compararemos los resultados hallados en los diferentes campos de estudio analizados.

\section{Análisis de resultados y conclusiones}

A partir del análisis realizado pudimos identificar diferentes concepciones acerca del valor de lo vivo en las distintas áreas de la biología indagadas. Resumimos los resultados obtenidos en la siguiente tabla.

\begin{tabular}{|c|c|c|c|}
\hline $\begin{array}{c}\text { Tipo de valoración/ } \\
\text { Área de estudio }\end{array}$ & Valor productivo & Valor gnoseológico & Valor intrínseco \\
\hline $\begin{array}{c}\text { Biología molecular y } \\
\text { genética }\end{array}$ & + & + & - \\
\hline Fisiología & + & + & - \\
\hline $\begin{array}{c}\text { Biología de la } \\
\text { conservación }\end{array}$ & + & - & + \\
\hline
\end{tabular}

Resumen de las principales concepciones acerca del valor de lo vivo halladas en las diferentes áreas de estudio de la biología analizadas (el signo '+' indica la presencia del tipo de valoración referida; el signo '-' indica su omisión o negación).

\footnotetext{
${ }^{7}$ De hecho, estos autores definen directamente el valor intrínseco como "la importancia interna de una especie, sin referencia alguna a su utilidad para humanos u otras especies" (Hunter \& Gibbs 2007, p. 399).

${ }^{8}$ En este sentido podemos diferenciar entre la corriente ecocentrista (predominante en la biología de la conservación) y la perspectiva denominada biocentrismo, desde la cual se destaca el valor intrínseco de cada organismo (Lindenmayer \& Burgman 2005). El biocentrismo no constituye una perspectiva mayormente considerada en los libros de texto.
} 
Retomando el objetivo que hemos planteado, hallamos como principal elemento en común entre las diferentes subdisciplinas analizadas una importante presencia del valor productivo de lo vivo en todos los campos. Además, encontramos la omisión o negación del valor intrínseco en las subdisciplinas que abordan los niveles inferiores de organización (biología molecular, genética y fisiología). En conjunto ambos resultados parecen indicar que lo vivo es valorado principalmente por su utilidad. En relación con esta observación cabe mencionar que algunos autores han destacado la creciente importancia de los objetivos pragmáticos y utilitarios en la ciencia (Echeverría 2003, Linares 2008). Desde esta perspectiva, en algunos ámbitos científicos, el interés por manipular los objetos naturales y transformarlos en materia de producción estaría ganando preeminencia respecto del tradicional objetivo científico, mayormente teorético, de conocer y explicar el mundo natural. En este sentido, la importancia de la valoración productiva de lo vivo por sobre otros tipos de valor podría vincularse con la presencia de tales objetivos pragmáticos y utilitarios en algunas de las áreas que hemos indagado. En nuestro análisis, el campo de la genética pareciera ser el que más claramente expresa ese interés utilitario, dado que hemos encontrado ampliamente representada en esa área la valoración de lo vivo como materia prima y objeto de producción. A su vez, la importancia otorgada al valor gnoseológico de lo vivo, tanto en el campo de la genética como en el de la fisiología, podría ser interpretada como un indicio de la persistencia de los objetivos teoréticos en esos campos. Sin embargo, dado el carácter exploratorio del análisis que aquí presentamos (y las limitaciones mencionadas en la Sección 1), estos elementos deberán ser indagados con mayor profundidad en futuros trabajos.

Por otra parte, una de las principales diferencias halladas entre los campos de estudio analizados refiere a la valoración intrínseca de lo vivo, la cual solamente pareciera reconocerse en el caso de la biología de la conservación. Tal como desarrollamos, en este campo de estudio el valor intrínseco de la biodiversidad constituye uno de los principales fundamentos para su conservación. Retomando la hipótesis que hemos planteado, cabría suponer que este reconocimiento del valor intrínseco de lo vivo podría constituir un foco de conflicto entre la biología de la conservación y las áreas en las cuales los organismos sólo son valorados y utilizados como herramientas productivas y gnoseológicas. Por lo tanto cabe preguntarse cómo es posible que coexistan al seno de la biología estas dos visiones dispares y potencialmente conflictivas acerca del valor atribuido a lo vivo. Una primera respuesta a este interrogante podría referir a la fragmentación del organismo como objeto de estudio y a la creciente especialización y separación de los diferentes campos de investigación que conforman la biología. Sin embargo, a partir de nuestro análisis, podemos señalar otro elemento que puede contribuir a explicar tal coexistencia. Como hemos desarrollado, la biología de la conservación parece atribuir valor intrínseco fundamentalmente a entidades superiores al organismo, tales como especies y ecosistemas, a la vez que pareciera negar el valor intrínseco de los organismos individuales. De esta manera la atribución de valor intrínseco a ciertas entidades biológicas distintas de los organismos individuales en el campo de la biología de la conservación, no entra en conflicto con la valoración utilitaria de los organismos en otros campos de la biología, ni con su utilización como herramientas productivas. Por lo tanto, respondiendo a la hipótesis que hemos planteado en la Sección 1, la negación de valor intrínseco a los organismos contribuiría a evitar posibles conflictos o contradicciones éticas entre las subdisciplinas biológicas que hemos analizado.

Para finalizar, cabe mencionar que la negación del valor intrínseco de los organismos, sumada a la importancia otorgada a los aspectos productivos e instrumentales en los campos científicos que hemos analizado, deja abiertos diversos interrogantes acerca de los supuestos valorativos y las bases éticas subyacentes al estudio de lo vivo en la biología actual. Un importante desafío para la biología, en un contexto en el cual el acelerado desarrollo científico y tecnológico genera diversas controversias éticas, consiste en poner en cuestión los propios valores y supuestos que subyacen a las prácticas científicas. En este sentido, si bien reconocemos en la biología de la conservación cierta reflexión acerca del valor de lo vivo, este tipo de consideraciones no parece extenderse a otras áreas de la biología. La filosofía de la ciencia puede entonces contribuir al desarrollo de reflexiones éticas poniendo de relieve los valores 
subyacentes a la ciencia como primer paso para su puesta en discusión, aportando así a la construcción de prácticas científicas que puedan asumir más cabalmente su dimensión ética.

Bibliografía

Alberts, B., Johnson, A., Lewis, J., Raff, M., Roberts, K. y P. Walter (2007), Molecular Biology of the Cell, $5^{\text {th }}$ edition, New York: Garland Science.

Barbero, S. (2014), La relación entre la neurobiología y la medicina. Un análisis del caso del trastorno por déficit de atención con hiperactividad (ADHD) desde la filosofía de la biología, Tesis de Licenciatura, Buenos Aires: Universidad de Buenos Aires.

Boron, W.F. y E.L. Boulpaep (2012), Medical Physiology. A Cellular and Molecular Approach, $2^{\text {nd }}$ edition, Philadelphia: Saunders.

Bunner, A.E., Chandrasekera, P.C. y N.D. Barnard (2014), "Knockout Mouse Models of Insulin Signaling: Relevance Past and Future", World Journal Diabetes 5(2): 146-59.

Callicott, J.B. (1990), "Whither Conservation Ethics?", Conservation Biology 4: 15-20.

Chakravartty, A. (2015), "Scientific Realism", The Stanford Encyclopedia of Philosophy. Accesible en: http://plato.stanford.edu/archives/fall2015/entries/scientific-realism/.

de Boo, J. y A. Knight (2008), "Increasing the Implementation of Alternatives to Laboratory Animal Use", Alternatives to Animal Testing and Experimentation 13(3): 109-117.

de Martínez, C.A.C., de Osorio, A.M., Martínez, C., Yunta, E.R. y F.L. Stepke (2007), El animal como sujeto experimenta. Aspectos técnicos y éticos, Santiago de Chile: CIEB, Universidad de Chile.

Echeverría, J. (2003), La revolución tecnocientifica, Madrid: Fondo de Cultura Económica.

Elliott, K.C. y D.J. McKaughan (2014), "Nonepistemic Values and the Multiple Goals of Science”, Philosophy of Science 81(1): $1-21$

Fernández Chaves, F. (2002), "El análisis de contenido como ayuda metodológica para la investigación”, Ciencias sociales 2(96): 33-54.

García Palacios, E.M., Galbarte, J.C.G., Cerezo, J.A.L., Luján, J.L., Gordillo, M.M., Osorio, C. y C. Valdés (2001), Ciencia, Tecnología y Sociedad: una aproximación conceptual, Madrid: Organización de Estados Iberoamericanos para la Educación, la Ciencia y la Cultura.

Griffiths, A.J.F., Wessler, S.R., Carroll, S.B. y J. Doebley (2010), Introduction to Genetic Analysis, $10^{\text {th }}$ edition, New York: WH Freeman.

Groom, M.J., Meffe, G.K. y R. Carrol (2006), Principles of Conservation Biology, $3^{\text {rd }}$ edition, Sunderland, MA: Sinauer Associates.

Gudynas, E. (2009), El mandato ecológico. Derechos de la Naturaleza y políticas ambientales en la nueva Constitución, Quito: AbyaYala.

Gudynas, E. (2015), Derechos de la Naturaleza, Buenos Aires: Tinta Limón.

Heler, M. (1996), Ética y ciencia: la responsabilidad del martillo, Buenos Aires: Biblos.

Hickman, C.P., Roberts, L.S. y A. Larsen (2006), Principios integrales de Zoología, 13a edición, Madrid: McGraw-Hill Interamericana.

Hunter, M. y J. Gibbs (2007), Fundamentals of Conservation Biology, Oxford: Blackwell Publishing.

Kincaid, H., Dupré, J. y A. Wylie (eds.) (2007), Value-Free Science: Ideals and Illusions?, Oxford: Oxford University Press.

Klug, W.S., Cummings, M.R., Spencer, C.A. y M.A. Palladino (2012), Concepts of Genetics, $10^{\text {th }}$ edition, San Francisco: Pearson Education Inc.

Lewin, B. (1999), Genes VII, $7^{\text {th }}$ edition, Oxford: Oxford University Press. 
Linares, J.E. (2008), Ética y mundo tecnológico, México: Fondo de Cultura Económica.

Lindenmayer, D. y M. Burgman (2005), Practical Conservation Biology, Collingwood: CSIRO Publishing.

López Noguero, F. (2002), “El análisis de contenido como método de investigación”, En clave pedagógica 4: 167-180.

Maingueneau, D. (1996), "El ethos y la voz de lo escrito", Versión 6: 79-92.

Marcuse, H. (1985), El hombre unidimensional. Ensayo sobre la ideología de la sociedad industrial avanzada, Barcelona: Planeta-Agostini.

Piñuel, J. (2002), "Epistemología, metodología y técnicas de análisis de contenido", Estudios de Sociolingüística 3(1): 142.

Purves, D., Augustine, G.J., Fitzpatrick, D., Hall, W.C., LaMantia, A.S. y L.E. White (2012), Neuroscience, $5^{\text {th }}$ edition, Sunderland, MA: Sinauer Associates Inc.

Randall, D., Burggren, W.W. y K. French (2001), Eckert Animal Physiology: Mechanisms and Adaptations, $5^{\text {th }}$ edition, New York: W. H. Freeman.

Reiss, J. y J. Sprenger (2014), "Scientific Objectivity", The Stanford Encyclopedia of Philosophy. Accesible en: http://plato.stanford.edu/archives/fall2014/entries/scientific-objectivity/.

Rodríguez Alcázar, F.J. (1997), "Esencialismo y neutralidad científica”, en Rodríguez Alcázar, F.J., Medina Doménech, R.M. y J. Sánchez Cazorla (eds.), Ciencia, tecnología y sociedad: Contribuciones para una cultura de paz, Granada: Editorial Universidad de Granada, pp. 49-84.

Taylor, P.W. (1986), Respect for Nature: A Theory of Environmental Ethics, Princeton: Princeton University Press.

Van Dyke, F. (2008), Conservation Biology. Foundation, concepts, applications, $2^{\text {nd }}$ edition, New York: Springer. 